\title{
Study on Multi-path Low-light Stress Reliability Test System ZHANG Lei ${ }^{\mathrm{a}}$, WANG Aiguo \\ Changchun University of Science and Technology, Changchun 130022, Jilin, China \\ a benchi4500@126.com
}

Keywords: Low-light level night vision device; integrating sphere; low-light level stress; reliability test

\begin{abstract}
During the design approval and production approval tests on the low-light night version device, the light stress reliability test must be done. In this paper, the author analyzed the actual operational environment of the low-light night version device and designed a multi-path low-light reliability test system with adjustable illumination from two aspects including natural light spectrum and illumination in the night sky. Four-path combined integrating sphere low-light stress systems are adopted to simulate nighttime as the illumination quantificationally. During the test, the programs are designed according to the test so as to exert variable light stresses on the low light (namely product) automatically, monitor and control the light stress level automatically, identify and record the faults of the low-light night version device automatically, which soundly revolve the demands of the light stress reliability test of the low-light night version device.
\end{abstract}

\section{Foreword}

The low-light night version device is a photoelectric apparatus based on image intensifier. It has a low random failure rate within the valid lifetime, but its performance can fall and be void with the increase of time[1]. During operation, it is easy to result in the image faults of the low-light night version device due to low-light environment mutation and light stress shock, which can influence the operation. Therefore, during the design approval and production approval tests on the low-light night version device, the light stress reliability test must be conducted. At present, as for the light stress reliability test, firstly, the outdoor natural nighttime environment is adopted directly, which is greatly influenced by ambient; secondly, it can be conducted through simulation of low-light environment in a dark room, through which the covered space is larger and it is not convenient for the operator to record. It is assumed that the multi-path low-light stress system adopts double integrating sphere low-light sources. design the illumination-adjustable integrating sphere type low-light stress system and realize the simulation of nighttime as the illumination quantificationally. During the test, the programs can be designed according to the test so as to exert variable light stresses. automatically, monitor and control the light stress level automatically, identify and record the faults of the low-light night version device automatically, which soundly resolve the demands of light stress reliability test of the low-light night version device.

\section{Composition and principle of low-light stress system}

The system is composed of integrating sphere type light source, collimator, CCD camera, computer management system and so on. The low-light night version device is provided with a low-light objective environment required by the reliability test so as to monitor and control the luminous intensity of the light source and the exerted light stress level continuously, supervise and estimate the work status and image quality of the low-light night version device. As for the composition of the system, refer to Figure 2-1. 


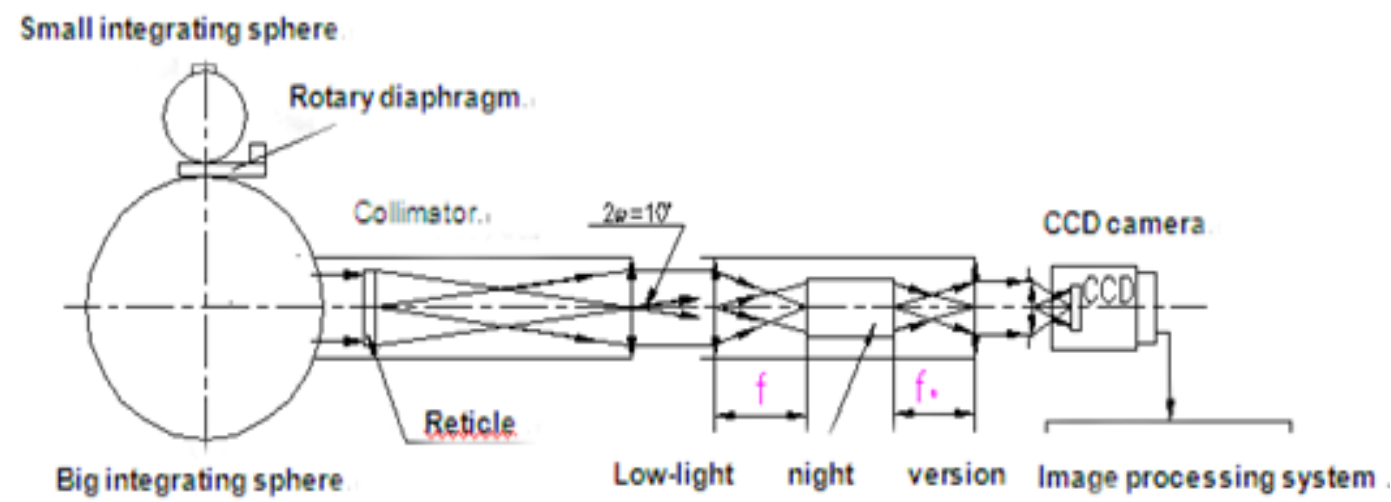

Figure 2-1: Composition of branch light stress system

The integrating sphere type light source adopts a double integrating-sphere structure, which consists of a big integrating sphere and a small integrating sphere. The small integrating sphere part is also named as lamp house system, the outlet of the small integrating sphere has a butt joint with the big one, so that the specific light energy diffused from the small integrating sphere can enter the big one through the outlet and then continue the diffuse reflection uniformly. The uniform low-light illumination required is given off from the outlet of the big integrating sphere. The big integrating sphere is designed with four outlets which are jointed with the object lens image planes (focal planes) of four collimators, then the standard low-light sources simulated can be obtained at the exit-pupil place of the collimators (see Figure 2-2).

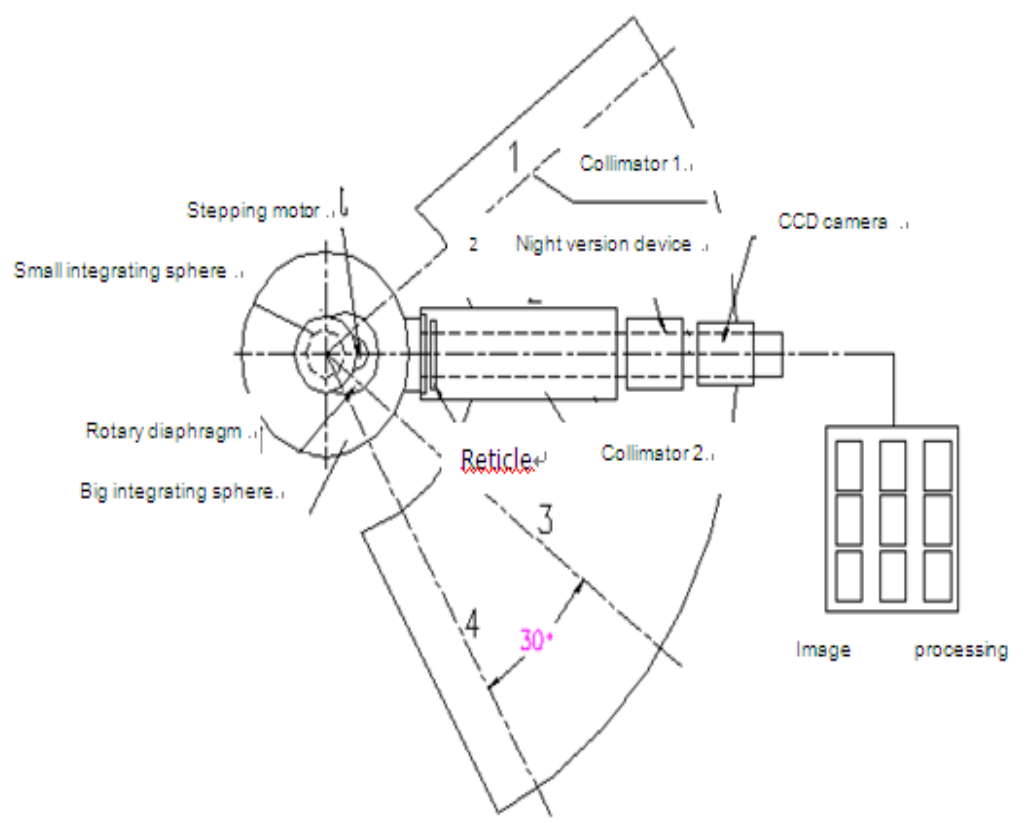

Figure 2-2: General layout of branch light stress system

The low-light illumination can be realized through switching the variable diaphragms between small and big integrating spheres. The diaphragm adopts a rotary-disc structure, there are circular holes with different illuminations calibrated, which are distributed equally on the circumference of the rotary disc, and a computer is used to control the running of the rotary disc so as to realize the switching of different illuminations. The low-light objective adopts a projection-type low-light resolution chart which is placed at the entrance-pupil place of the collimator lens, to provide measurement objectives for the low-light night version device.

\section{Calculation of the integrating sphere illumination}

Window illumination of the small integrating sphere.

The small integrating sphere internal walls paints the reflection coating with the neutral spectra 
reflectance, in addition to ground-glass windows inside the ball no other absorption light foreign matter. The window illumination of the light source wall consists of two parts: the illumination $E_{0}$ of direct light and light irradiate the ball-wall points multiple reflections to the window illumination $E_{i}$. That is:

$$
\begin{aligned}
& E_{\min }=E_{0}+E_{1}+E_{2}+\cdots E_{n}+\cdots \\
& =\frac{\phi}{4 \pi r^{2}}+E_{1}+\rho(1-f) E_{1}+\cdots[\rho(1-f)]^{n} E_{1}+\cdots \\
& =\frac{\phi}{4 \pi r^{2}}+E_{1}+\frac{\rho(1-f)}{1-\rho(1-f)} E_{1} \\
& =\frac{\phi}{4 \pi r^{2}}+\frac{1}{1-\rho(1-f)} E_{1} \\
& =\frac{\phi}{4 \pi r^{2}}+\frac{\rho \phi}{4 \pi r^{2}} \cdot \frac{1}{1-\rho(1-f)}
\end{aligned}
$$

The small integrating sphere radius $\mathrm{r}=150 \mathrm{~mm}$, the sphere wall reflectance ratio $\rho=0.8$. The calibration lamp's luminous flux $\Phi=630 \mathrm{Lm}$, the window radius $r_{0}=50 \mathrm{~mm}$. The opening ratio of the small integrating sphere is:

$$
f=\frac{S_{\text {球冠 }}}{S_{\text {积分球 }}}=\frac{2 \pi r h}{4 \pi r^{2}}=\frac{h}{2 r} \quad h=r-\sqrt{r^{2}-r_{0}^{2}}
$$

Inside: $r$-the small integrating sphere radius, $r_{0}$-spherical cap radius, $h$ - spherical cap height, $f=0.028$

$$
\begin{aligned}
& E_{\text {min }}=\frac{630}{4 \pi(0.15)^{2}}+\frac{0.8 \times 630}{4 \pi(0.15)^{2}} \times \frac{1}{1-0.8 \times(1-0.028)}=10242(l x) \\
& f=\frac{S_{\text {球冠 }}}{S_{\text {积分球 }}}=\frac{2 \pi r h}{4 \pi r^{2}}=\frac{h}{2 r}
\end{aligned}
$$

the appropriate flux $\phi_{0}$ of the small integrating sphere window is

$$
\phi_{0}=E S=10240 \times \pi \times(0.05)^{2}=80.4(\mathrm{~lm})
$$

\section{Large integrating sphere illumination.}

After the emergent light from the small integrating sphere pass the frosted glass, they become diffuse transmittance light, and in accordance with the radiation cosine distribution, the exitance $\mathrm{M}$ should be approximately equal to the value of illumination E. After the ground-glass' light absorption and light scattering are ignored, and go through the transmission board with transmission ratio $\tau_{0}$, the luminous flux of re-entering the large integrating sphere is:

$$
\phi_{0}^{\prime}=\tau_{0} E S=80.4 \tau_{0}(\mathrm{~lm})
$$

As the diffuse transmittance light entrances from the integrating sphere above openings, while the large integrating sphere's four exits distribute along the circumference of its equator, so that the exit illumination are all a result of the ball-wall points multiple reflections to the exit illumination sum, that is

$$
\begin{aligned}
& E_{\max }=E_{1}+E_{2}+\cdots+E_{n}+\cdots \\
& =E_{1}\left(1+\bar{\rho}+\bar{\rho}^{2}+\cdots+\bar{\rho}^{n}+\cdots\right) \\
& =\frac{\phi_{0}^{\prime}}{4 \pi R^{2}} \cdot \frac{\rho}{1-\bar{\rho}}
\end{aligned}
$$

The large integrating sphere radius $\mathrm{R}=500 \mathrm{~mm}$, integrating sphere exit radius $\mathrm{R}_{0}=100 \mathrm{~mm}$, the large integrating sphere wall reflectance ratio $\rho=0.8$. 


$$
f=\frac{S_{\text {球冠 }}}{S_{\text {积分球 }}}=\frac{2 \pi R H}{4 \pi R^{2}}=\frac{H}{2 R}=0.01
$$

The large integrating sphere has four openings, and the openings' reflection ratio is 0 . So the average reflectance ratio of the large integrating sphere wall is:

$\bar{\rho}=\rho(1-4 f)=0.8(1-4 \times 0.01)=0.768$

Get:

$$
E_{\max }=\frac{80.4 \tau_{0}}{4 \pi \times 0.5^{2}} \times \frac{1}{1-0.768}=110 \tau_{0}(l x)
$$

\section{Calculation of transmittance.}

Since the exit of large integrating sphere docks with the collimator image surface, the light changes diffuse transmitted light through the frosted glass, and the luminous exitance $M \approx E_{0}$. Thus within the solid angle from the collimator image surface center to objective aperture, the flux as follows:

$$
\varphi_{p}=M S \cdot \sin ^{2} u
$$

According to the corresponding parameters of collimator, focal length $\mathrm{f}^{\prime}=960 \mathrm{~mm}$, diameter $\mathrm{D}=$ $120 \mathrm{~mm}$, angel of view $2 \omega=10^{\circ}$. The effective area of the collimator image surface can be got.

$$
\begin{aligned}
& S=\pi\left(0.960 \times \operatorname{tg} 5^{0}\right)^{2}=\pi \times(0.084)^{2}=0.022 \mathrm{~m}^{2} ; \\
& \sin u=\sin [\operatorname{arctg}(60 / 960)]=0.062
\end{aligned}
$$

Substitute the above values into the equation (3.5), get

$$
\varphi_{p}=110 \tau_{0} \times 0.022 \times(0.062)^{2}=0.0092 \tau_{0}(\mathrm{~lm})
$$

As the transmission coefficient of the collimator objective $\tau \approx 70 \%$, then get the illumination of collimator objective exit pupil as follows:

$$
E_{p}=\varphi_{p} \tau / S=0.011 \tau_{0} \tau / \pi \times(0.06)^{2}=0.68 \tau_{0}(\mathrm{Lx})
$$

If the illumination ask $E_{p}=1 \times 10^{-1}(\mathrm{Lx})$ in the tested low-light sight objective, by equation (3-6) it amounts to the first transmission board's transmittance $\tau_{01}$ as follows:

$$
\tau_{01}=0.1 / 0.68=14.7 \% \text {; }
$$

For the same reason, the transmittance $\tau_{02}$ of the second transmission board is counted as follows:

$\tau_{02}=0.01 / 0.68=1.475 \%$

The third and fourth transmission boards' transmittance is still to take $\tau_{02}=1.47 \%$ too, only the transmittance boards clear area are reduced to the original 1/10,1/100, it can get four kinds of light stress sources that the illumination is respective $1 \times 10^{-1}, 1 \times 10^{-2}, 1 \times 10^{-3}, 1 \times 10^{-4}$.

Integrating sphere illumination on the above theoretical calculation can be used as an important basis for the design. In fact, because the light of the sources is non-uniform in all directions, the openings of the large and small integrating sphere, obscuration, the neutral coating on neutral wall are non-uniform, the actual deviation ratio of reflectance, the deviation of objective transmittance and so on, the final transmittance $\tau_{0}$ of the transmittance board and transmittance area are selected in accordance with the actual situation, in order to fully meet the tested sight needs for the different illumination.

\section{Reliability test on Low-light level night vision device}

\section{Collimator exit pupil illumination.}

In the standard darkroom, four collimators exit pupil (low-light level night vision device object lens entrance pupil) illumination, illumination non-uniformity and consistency of four illuminations are respectively tested by BG-2 micro-light luminometer, test results the following table. 
Sheet 1-1 Collimator illumination test data sheet

\begin{tabular}{|c|c|c|c|c|}
\hline Illumination level & $1^{\#}$ Collimator & $2^{\#}$ Collimator & $3^{\#}$ Collimator & $4^{\#}$ Collimator \\
\hline $1 \times 10^{-1}$ & 0.92 & 1.10 & 1.01 & 1.09 \\
\hline $1 \times 10^{-2}$ & 1.02 & 1.22 & 1.10 & 1.16 \\
\hline $1 \times 10^{-3}$ & 1.08 & 1.28 & 1.16 & 1.23 \\
\hline $1 \times 10^{-4}$ & 1.56 & 2.06 & 1.76 & 1.85 \\
\hline
\end{tabular}

It can be seen from the test results that the collimator export illumination and the non-uniformity error is very small, the greater illumination leads to the smaller the error. Four collimators consistency is better able to meet low-light level night vision device product test requirements very well.

\section{Light stress test on low-light night version device.}

The low-light stress system above is adopted to carry out continuous reliability tests on the low-light night version device, which verifies the feasibility of the system from engineering. The low-light stress adopts the low-light sources consisting of integrating spheres and collimators above, the low-light night version device adopts a low-light sighting piece with type of $\mathrm{XX}$, the image acquisition is realized through an industrial interface 1394 black and white CCD camera with type of MV-VS142FM/FC, the highest resolution totals 1392x1040. A computer is used to control the switching of the light stresses, continuously monitor the images of the low-light night version device, automatically identify and record the fault images of the low-light night version device.

The test adopts 4 night version devices working simultaneously for continuous $600 \mathrm{~h}$, which realizes the automatic switching of light stresses, automatic monitoring on images and automatic records of fault images, saves manpower, reduces the labor intensity and raises the accuracy and reliability of the test. The fault images monitored and recorded during test are available as follows:

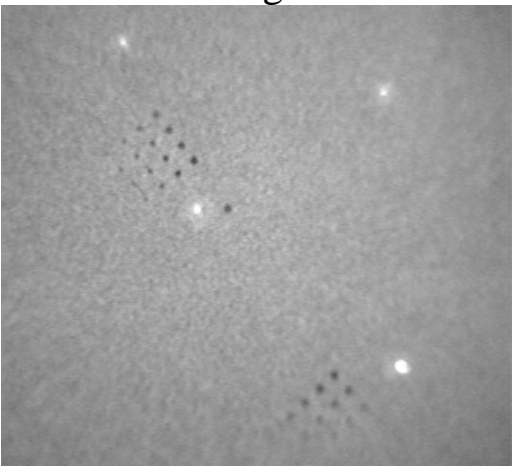

(a) Fault image with bright spots

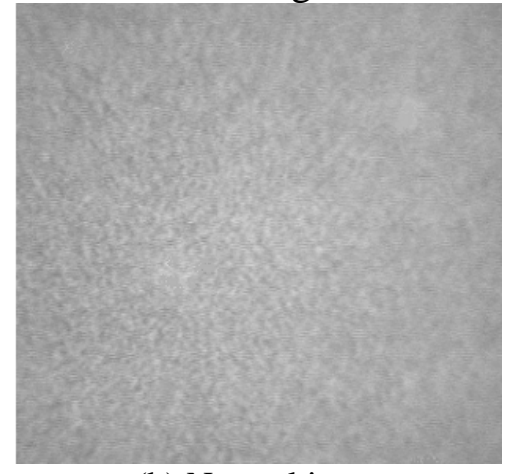

(b) Normal image

Figure 3: Comparison of night version device images

\section{Conclusion}

Through the exit-pupil illumination inspection and the simulation comparison test on the integrating sphere type low-light stress system, it proves that the system can simulate the low-light environment quantificationally. The multi-path low-light stress sources provided can meet the requirements of the light stress reliability test of the low-light night version device. The system adopts a building block type structure and uses the same integrating sphere light source exerting the light stresses on four groups of test systems simultaneously, which can ensure the consistency of the light stress sources and be convenient for transverse comparison of four groups of test results. The light source is characterized of high utilization and sound screening performance, which can reduce the influences of the outside error sources effectively and raise the objectivity and reliability of the test results evidently. Four groups of test systems can be used simultaneously and also separately, which is convenient for maintenance or renewal of components.

\section{References}


[1] WANG Gao-ming,GUO Cheng:Simulation of the LightEnergy Uniform ity of IntegratingSphere Based onMonte CarloMethod, LASER\&INFRARED, 2009,39(1), 67-69

[2] YE Yuanlong, WU Xiaorong: Simulationg of Low-lght-level Condition in laboratory, LASER\&INFRARED, 2005,35(12), 942-943

[3] SHI Jifang, LIU Yu: Night Sky Radiation Simulation for Test ofLow-Level-Light Night Vistion Systems, JOURNAL OF APPLIED OPTICS, 2003,24(5), 11-13

[4] CHEN Yingping: The Analysis and Study on the Reliability of Chinese Military Night Viewing Instrument, OPTOELECTRONIC TECHNOLOGY, 2003,23(1), 68-70

[5] Yang Linhua,Jian Dayuan: Research of Integrating Sphere Solar Simulating Source,Spacecraft Environment Engineering,Vol. 2004.22 (2) ,116-119

[6] CAI Kejun, FU Yuegang: Night Simulation System for Low-Level-Light Night Vision Systems Testing, Infrared Technology, Vol. 2007.29(2) ,102-106 